\title{
Curing behaviour of epoxidized soybean oil with biobased dicarboxylic acids
}

Run-Tao Zeng, Ying Wu, Yi-Dong Li*, Ming Wang, Jian-Bing Zeng*

School of Chemistry and Chemical Engineering, Southwest University, Chongqing 400715, China

Email:dongdongdong555@sina.com (Y.D. Li) and jbzeng@swu.edu.cn (J. B. Zeng) fax/tel: $+86-23-68254000$. 
Abstract: In this study, we report the curing of ESO with biobased dicarboxylic acids (DCAs) with different carbon chain-lengths to synthesize fully sustainable polymers. Both non-isothermal and isothermal curing processes analysis indicated that the curing rate and activation energy decreased with increasing chain-length of DCAs. The optimum -COOH/epoxy molar ratio is 0.7 for preparation of ESO/DCA cured product with maximum degree of crosslinking. Addition of 4-N, N-dimethylaminopyridine (DMAP) as a catalyst can efficiently accelerate the curing rate and reduce activation energy. We systemtically studied the effect of chain-length of DCAs on the physical properties of cured products, and found that with increase in chain-length of DCAs, the glass transition temperature of the cured ESO/DCA decreased, the tensile strength and Young's modulus increased while elongation at break decreased, due to the decreased crosslinking density resulted from the increased chain-length between crosslinking sites. All cured ESO/DCA showed excellent thermal stability with initial decomposition temperature of higher than $340{ }^{\circ} \mathrm{C}$.

Keywords: Epoxidized soybean oil; Dicarboxyl acid; Curing behavior 


\section{Introduction}

In recent years, sustainable polymers, derived from renewable resources, have attracted or renewed significant interests due to the increased resource crisis and environmental concerns associated with petroleum-based polymers ${ }^{1-5}$. Plant oils, as triglycerides composed of various fatty acids depending on the plant and the growing conditions, are regarded as ideal renewable feedstocks for fabricating various sustainable polymers with different structures, properties and application, since plant oils with different origins contain different functional groups, such as $\mathrm{C}=\mathrm{C}$ double bonds, ester groups, and hydroxyl groups, available for various chemical reactions towards fabrication of different polymers including polyesters, polyamides, polyurethanes, epoxy resins, etc ${ }^{6,7}$. Soybean oil is a major plant oil which has found many applications in soaps, paints, coatings, lubricants, and bioplastics since nineties of last century ${ }^{1}$.

The abundant unsaturated $\mathrm{C}=\mathrm{C}$ double bonds within the constituent fatty acids of soybean oil facilitate the production of epoxidized soybean oil (ESO) via epoxidation reaction $^{8}$. ESO finds significant utilizations in material science and industry. It is widely used as green plasticizer for various polymers like poly(vinyl chloride) ${ }^{9}$, poly(lactic acid $)^{10}$ and ethyl cellulose ${ }^{11}$. The presence of reactive epoxy groups enables direct synthesis of epoxy resin from ESO via ring opening reaction with amino or carboxyl groups containing compounds such as diamine ${ }^{1}$, dicarboxylic $\operatorname{acid}^{12}$ and anhydride ${ }^{13}$. In corporation of high reactive double bond by reacting with acrylic acid or allyl alcohol provides an efficient way for ESO to produce polymers 
via free radical polymerization ${ }^{14-16}$. In addition, suitable chemical reactions are able to transform ESO to polyols towards fabrication of polyurethanes ${ }^{17-19}$.

Carboxylic acids and anhydrides are the second most important curing agents for epoxy resins followed amines. Some aliphatic dicarboxylic acids (DCAs) such as adipic acid, sebacic acid and 1,12-dodecandicarboxylic acid can be produced via biomass-based routes ${ }^{20}$. Therefore, the use of those DCAs as curing agents would promote the sustainable development of epoxy resins. Sporadic studies have reported the curing of ESO with dicarboxylic acids or anhydride ${ }^{12,21,22}$. Few literatures studied the curing behaviors of other epoxidized vegetable oils with dicarboxylic acids ${ }^{20}$. ESO is the most abundant and commercialized epoxidized vegetable oil. However, there is lacking of comprehensive literature that reports the use of biobased DCAs as curing agents for ESO to form fully sustainable polymers and reveals the curing behavior and the physical properties of the resultant sustainable polymers. Thus, it is necessary to investigate the effect of biobased DCAs as curing agents on the curing process of ESO and the properties of the resultant curing products, from the viewpoint of developing novel sustainable polymers from plant oils.

In this study, we investigate the curing of ESO and biomass-derived DCAs systematically, reveal the effect of chain-length of DCAs on the curing behavior of the reaction between ESO and DCAs in the absence/presence of a catalyst, and report the effect of the chain-length of DCAs and molar ratio of ESO to DCA on the glass transition, thermal stability, and mechanical properties of the obtained polymers. 


\section{Experimental section}

\subsection{Materials}

ESO with epoxy value of $\geq 6.0 \%$ was purchased from Micxy Chemical Co., Ltd (Chengdu, China). The average number of epoxy group per an ESO molecule is 4.1 as calculated by NMR analysis. Adipic acid (AA, 99\%), sebacic acid (SA, 98.5\%), 1,12-dodecandicarboxylic acid (DA, 99\%) and 4-N, N-dimethylaminopyridine (DMAP, 99\%) were obtained from the Micxy Chemical Co., Ltd. All reactants and catalyst were used as received. Dichloromethane with AR grade was procured from Kelong chemical reagent factory (Chengdu China).

\subsection{Non-isothermal curing of ESO with DCAs}

Non-isothermal curing of ESO with DCAs were investigated by differential scanning calorimetry (DSC) with a NETZSCH instruments DSC-214. Predetermined amount of ESO and DCA with/without certain amount of DMAP were first mechanically stirred at room temperature for $10 \mathrm{~min}$ to obtain uniform mixtures. The total weight of the mixtures was $10 \mathrm{~g}$. Then, 7 9 $\mathrm{mg}$ mixtures were placed in an aluminum DSC pan, which were then heated from $25{ }^{\circ} \mathrm{C}$ to $300{ }^{\circ} \mathrm{C}$ at a heating rate of $10{ }^{\circ} \mathrm{C} / \mathrm{min}$ to study the effect of catalyst content and molar ratio of -COOH/epoxy on the curing of ESO with DCAs. Three runs were performed for each sample and the averaged results were reported. To study the effect of chain-length of DCA on the curing behavior of ESO, we recorded the DSC heating scans of mixtures of ESO with different DCAs with the stoichiometric relationship, $\mathrm{R}$, of 0.7 ( $\mathrm{R}=$ acid groups/epoxy groups). To investigate the curing activation energy, dynamic DSC runs for the sample were performed under 
4 different heating rates of $2.5,5,10$ and $20{ }^{\circ} \mathrm{C} / \mathrm{min}$ in the temperature range of $25 \sim 300{ }^{\circ} \mathrm{C}$. The experiment was carried out under $N_{2}$ atmosphere with a constant flow of $50 \mathrm{~mL} \mathrm{~min}^{-1}$.

\subsection{Isothermal curing of ESO with DCAs}

Isothermal curing of ESO with DCAs were carried out in a single-neck round-bottomed flask with a magnetic stirrer. Predetermined amount of ESO and DCA with/without certain amount of DMAP were added into the flask. The mixtures were stirred and cured at predetermined temperature. A part of product was taken out at a given time to measure gel fraction. The product of $\sim 2 \mathrm{~g}$ was immersed in chloroform for 1 week at room temperature. The undissolved part was obtained by filtering and then vacuum dried at $60{ }^{\circ} \mathrm{C}$ for $24 \mathrm{hr}$. The gel fraction of the sample was calculated by the equation

$$
\text { Gel fraction }=\frac{\mathrm{W}_{i}-\mathrm{W}_{g}}{W_{i}} \times 100 \%
$$

where $W_{\mathrm{i}}$ is the initial weight and $W_{\mathrm{g}}$ is the dry weight of undissolved part of the cured sample.

\subsection{Sample sheets preparation}

The sample sheets of various DCAs cured ESO were prepared in a fan-assisted oven. The mixtures of ESO, DCA and DMAP were mechanically stirred at room temperature for $10 \mathrm{~min}$, and then poured into a Teflon pan with internal size of $100 \times 100 \times 5 \mathrm{~mm}^{3}$ and cured in the oven at $160{ }^{\circ} \mathrm{C}$ for 2 hours.

\subsection{Characterizations}

The FT-IR spectra of the samples were recorded on a RF-5301PC spectrophotometer 
(Shimadzu, Japan ) in a range of wavenumbers from 4000 to $400 \mathrm{~cm}^{-1}$. The resolution and scanning time were $4 \mathrm{~cm}^{-1}$ and 32 times, respectively.

The glass transition temperature of the completely cured sample with analyzed by a NETZSCH instruments DSC-214. 7 9 mg sample in an aluminum DSC pan was first cooled to $-50{ }^{\circ} \mathrm{C}$, after kept at this temperature for $3 \mathrm{~min}$, the sample was then heated to $50{ }^{\circ} \mathrm{C}$ at a heating rate of $10{ }^{\circ} \mathrm{C} / \mathrm{min}$. The heating scan was recorded for analysis.

The mechanical properties of the cured samples were measured with a Sansi Universal Testing Machine (CMT6503) according to at a crosshead speed of 5 $\mathrm{mm} / \mathrm{min}$ at room temperature. Dumb-bell shaped specimens with width and thickness of 4 and $1 \mathrm{~mm}$ were used for the test. The length between the two mechanical grips of the testing machine was $25 \mathrm{~mm}$. At least five specimens were tested for each sample, and the averaged result was reported.

Thermal stability of the cured sample under $\mathrm{N}_{2}$ atmosphere was performed on a TA TGA-Q600 from room temperature to $550{ }^{\circ} \mathrm{C}$ at a heating rate of $10{ }^{\circ} \mathrm{C} / \mathrm{min}$.

\section{Results and discussion}

\subsection{Non-isothermal curing behavior of ESO/DCA systems}

It is well known that the main reaction for the process of DCA cured epoxy is the ring opening of epoxy by attacking of carboxyl groups to form new ester and hydroxyl groups. As ESO contains more than two epoxy groups within a molecule, the curing of ESO and DCA must form crosslinking product, which may also contain some branches depending on the feed ratio of the reactants and the reaction degree. Fig. 1 
shows the proposed possible reaction between ESO and DCA, the chemical structure of cured product, as well as the digital photo of the transparent cured film.

The effect of chain-length of DCA on the non-isothermal curing behavior of ESO were first studied with $\mathrm{R}$ of 0.7 , as this ratio was reported to be optimum for some carboxylic cured other epoxidized plant oil system ${ }^{23}$. Fig. 2 shows the DSC heating scans of the cured systems at a heating rate of $10^{\circ} \mathrm{C} / \mathrm{min}$ in the range of $25 \sim 300{ }^{\circ} \mathrm{C}$. An endothermic peak at low temperature range $\left(<150{ }^{\circ} \mathrm{C}\right)$ was first observed for all samples, which was ascribed to the melt of the corresponding DCA. Then, a broad exothermic peak resulted from the curing reaction between ESO and DCA was observed. Regarding to the chain-length of DCA, it is obvious that exothermic peak shifted to higher temperature range with increasing chain-length. The peak temperature $\left(T_{\mathrm{p}}\right)$ of the DSC exothermic peak is usually used as an indicator to describe the reactivity of compounds in a curing reaction, and lower $T_{\mathrm{p}}$ represents higher reactivity ${ }^{16}$. The $T_{\mathrm{p}}$ and the total enthalpy of curing $\Delta H_{\mathrm{c}}$ are listed in Table 1. The results indicated that the reactivity of DCA with ESO follows the order: AA $>$ SA $>$ DA, which is reasonable since shorter-chained DCA exhibit higher mobility. In addition, the $\Delta H_{\mathrm{c}}$ decreased with increasing chain-length of DCA, which was probably due to the lowering carboxyl density.

Although the $T_{\mathrm{p}}$ shifted with chain-length of DCA, the $T_{\mathrm{p}} \mathrm{s}$ for all the DCAs considered in this study are more than $200{ }^{\circ} \mathrm{C}$. Therefore, fast curing rate can only be achieved at high temperatures. It is not preferred for the sustainable polymers fabrication due to high energy-consuming. Addition of catalysts provides an efficient 
way of lowing curing activation energy and thus accelerating curing rate. We evaluated the effect of using DMAP as a catalyst on the curing process of ESO and DCA. In the first place, we studied the effect of DMAP's content on the curing behavior of the system by using SA as the typical DCA with addition of various contents of DMAP from 0.1 to $3 \mathrm{wt} \%$ of ESO. The R with 0.7 was also used in this case.

Fig. 3 shows the DSC heating scans of ESO/SA curing system with addition of different amount of DMAP. It is obvious that the curing exothermic peak narrowed significantly and the $T_{\mathrm{p}}$ shifted gradually to lower temperature range with increasing DMAP content. Narrowed exothermic peak indicates accelerated curing reaction rate, due to the fact that less time consumed with narrower peak at a given heating rate. Therefore, we can conclude that the curing rate increased gradually with increasing DMAP content. For example, as shown in Fig. 3 and Table 1, the $T_{\mathrm{p}}$ of ESO/SA without DMAP was $206.6{ }^{\circ} \mathrm{C}$ and the exothermic peak extended from $130{ }^{\circ} \mathrm{C}$ to $300{ }^{\circ} \mathrm{C}$. Addition of $0.5 \mathrm{wt} \%$ DMAP decreased the $T_{\mathrm{p}}$ significantly to $189.6{ }^{\circ} \mathrm{C}$ and enabled the curing to finish in the temperature range of $130 \sim 260{ }^{\circ} \mathrm{C}$. Further increasing DMAP content to $1.0 \mathrm{wt} \%, T_{\mathrm{p}}$ further declined to $178.2{ }^{\circ} \mathrm{C}$, and the exothermic peak narrowed to the range of $130 \sim 250{ }^{\circ} \mathrm{C}$. Although $T_{\mathrm{p}}$ decreased once again to $172.5{ }^{\circ} \mathrm{C}$ with increasing DMAP content up to $3.0 \mathrm{wt} \%$, the decreasing extent seemed insufficient compared to the increasing amount of DMAP. For $\Delta H_{\mathrm{c}}$, the values ware all in the range of 232 243 J/g with various DMAP's contents. It is noted that a small endothermic peak at $\sim 60{ }^{\circ} \mathrm{C}$ occurred with the content of DMAP increased to 
$1.0 \mathrm{wt} \%$ or higher, which may correspond to the melt of some intermediates of DMAP and ESO.

We also evaluated the effect of DMAP content on the curing behavior of ESO/AA and ESO/DA systems. For brevity, two DMAP contents, i.e., $0.5 \mathrm{wt} \%$ and $1.0 \mathrm{wt} \%$, were considered. Similar results are obtained with increasing DMAP content for the both systems, as shown in Table 1 . The $T_{\mathrm{p}}$ of ESO/AA without DMAP is $200.2{ }^{\circ} \mathrm{C}$, addition of 0.5 and $1.0 \mathrm{wt} \%$ DMAP decreased the $T_{\mathrm{p}}$ to 188.6 and $178.5{ }^{\circ} \mathrm{C}$, respectively; and the $T_{\mathrm{p}}$ of ESO/DA without DMAP is $209.6{ }^{\circ} \mathrm{C}$, addition of DMAP decreased $T_{\mathrm{p}}$ to 191.4 and $180.5^{\circ} \mathrm{C}$, respectively. In addition, it is found that ESO/DA system showed the largest decline in $T_{\mathrm{p}}$ with $1.0 \mathrm{wt} \%$ DMAP compared to ESO/AA and ESO/SA systems, possibly due to the highest original $T_{\mathrm{p}}$ of ESO/DA system without catalyst.

The ratio of $\mathrm{COOH} /$ Epoxy $(\mathrm{R})$ would also influence the curing behavior of DCA cured ESO system. Therefore, we studied the effect of R on the non-isothermal curing behavior of ESO/SA system in the presence of $1.0 \mathrm{wt} \%$ DMAP to determine the optimum R for the present system. Fig. 4 shows the DSC heating scans of ESO/SA curing system with R shifting from 0.5 to 1.0 and DMAP content of $1.0 \mathrm{wt} \%$.

It is noted that $T_{\mathrm{p}}$ showed slight downtrend with increasing $\mathrm{R}$. The values were $180.4,180.6,178.2,177.9,173.4$ and $174.0{ }^{\circ} \mathrm{C}$ for the curing systems with $\mathrm{R}$ of 0.5 , 0.6, 0.7, 0.8, 0.9 and 1.0, respectively, which indicated the curing rate increased with increasing $\mathrm{R}$, probably due to the increased concentration of $\mathrm{COOH}$ functional groups. What we focus more is the effect of $\mathrm{R}$ on the $\Delta H_{\mathrm{c}}$ of the curing systems. The values 
increased first and then decreased with increasing $\mathrm{R}$ and the maximum occurred with the $\mathrm{R}$ of 0.7 . The values for the six samples were $150.1,180.6,242.5,193.1,164.3$ and $153.2 \mathrm{~J} / \mathrm{g}$, respectively. The results indicated that the optimum $R$ value for ESO curing with DCA was 0.7 . The low $\Delta H_{\mathrm{c}}$ is attributed to the insufficient amount of $-\mathrm{COOH}$ when the $\mathrm{R}$ is less than 0.7. While when the $\mathrm{R}$ is more than 0.7 , some $-\mathrm{COOH}$ groups remain, due to steric effect of long alkyl chains of ESO which can hinder accessibility of the acid groups. Therefore, free DCA and/or perhaps dandling ends may be present in the resultant curing product if $R>0.7$. In the following sections, we maintained the $R$ at 0.7 for further investigation.

To further study the effect of chain-length of DCA and DMAP content on the curing behavior of ESO/DCA systems, we performed dynamic DSC for some samples with different heating rates from 2.5 to $20{ }^{\circ} \mathrm{C} / \mathrm{min}$ and determined the activation energy for the curing reaction via the Kissinger method. Fig. 5a shows the dynamic DSC heating scans of ESO/SA system with $1.0 \mathrm{wt} \%$ DMAP as a typical example. It is obvious that $T_{\mathrm{p}}$ increases with heating rate. Other samples show the similar dynamic DSC curves, and for brevity, the curves are not shown.

From the $T_{\mathrm{p}}$ values of different heating rates, the curing activation energy can be determined by the Kissinger method from the equation ${ }^{24}$ :

$$
\frac{d\left[\ln \left(\beta / T_{p}^{2}\right)\right]}{d\left(1 / T_{p}\right)}=-\frac{\Delta E_{a}}{\mathrm{R}}
$$

where $\beta$ is the heating rate, $\mathrm{R}$ is the universal gas constant, and $\Delta E_{a}$ is the curing activation energy. Plotting $\ln \left(\beta / T_{p}^{2}\right)$ versus $1 / T_{p}$ gives straight line, as shown in Fig. 
5b. The value of $\Delta E_{a}$ is derived from the slop of the straight line. The number in sample designation in Fig. 5b represents DMAP content. As shown in Table 1, for the effect of chain-length of DCA, ESO/AA system showed the lowest activation energy. But it is worth noting that the value is very close to those of ESO/SA and ESO/DA systems, probably due to the relatively small difference in chain-length of the DCAs. It is more interesting to study the effect of DMAP content on the activation energy of the curing systems. For the three systems with different DCAs, addition of DMAP obviously reduces curing activation energies, which decreases with increasing DMAP content. The decrement in the value of $\Delta E_{a}$ is in the range of $7.5 \sim 11.6 \mathrm{~kJ} / \mathrm{mol}$ for the three systems by addition of $1.0 \mathrm{wt} \%$ DMAP. The results indicate that DMAP is an efficient catalyst for DCA cured ESO system.

\subsection{Isothermal curing behavior of ESO/DCA systems}

Fabrication of DCA cured ESO sustainable polymers is conveniently performed under isothermal conditions. Therefore, we investigated the isothermal curing behavior of ESO/DCA systems by calculating gel fraction of samples obtained by curing at different temperatures for various times. The effect of temperature on the isothermal curing behavior of ESO/DCA was first investigated by using SA as a typical DCA. The samples containing 1.0 wt\% DMAP were cured at three temperatures i.e., 140, 160 and $180{ }^{\circ} \mathrm{C}$, for various times, and then were charged into chloroform and kept for 1 week to determine the gel fraction. Fig. 6a shows the development of gel fraction of cured ESO/SA/DMAP-1.0 with time at different isothermal curing 
temperatures. It is found that no gel formed in the initial stage of curing about 5 10 min depending on the temperature, which was due to the formation of soluble branched products. Thereafter, the gel fraction increased significantly with prolonging time. For the effect of temperature, the increment in gel fraction increased obviously with increasing temperature. For example, the gel fraction at 30 min was 20.7 by curing at $140{ }^{\circ} \mathrm{C}$ while increased significantly to $86.1 \%$ and $94.7 \%$ by curing at 160 and $180{ }^{\circ} \mathrm{C}$, respectively. After $30 \mathrm{~min}$, the gel fraction increased very slightly with time for curing at 160 and $180{ }^{\circ} \mathrm{C}$, while the gel fraction still increased almost linearly within the considered time. The gel fractions for curing at 140,160 and $180{ }^{\circ} \mathrm{C}$ for 120 min were $82.5 \%, 93.4 \%$ and $95.6 \%$, respectively.

In order to obtain a good balance between curing rate and difference in curing rate with respect to cure of ESO with different DCAs, we studied the effect of chain-lengths of DCA on the isothermal curing behavior of ESO/DCA systems at $160{ }^{\circ} \mathrm{C}$. Fig. $6 \mathrm{~b}$ shows the development of gel fraction of ESO cured with different DCAs with time at isothermal curing temperature of $160{ }^{\circ} \mathrm{C}$. It can be seen that the gel fraction increased with decreasing chain-length of DCA at a given time when the time is $\leq 30 \mathrm{~min}$, indicating shorter-chained DCA shows faster isothermal curing rate, which is in accordance with the change of $T_{\mathrm{p}}$ versus chain-length of DCA obtained by dynamic DSC analysis. When the isothermal curing time prolonged to $120 \mathrm{~min}$, the gel fraction of all sample increased to around $92 \%$, which was indicative of almost fully curing. The presence of catalyst and some impurities resulted in the final gel fraction less than $100 \%$. 
For comparison, we carried out isothermal curing of ESO/SA in the absence of DMAP, and found that more than 8 hours were required for the sample to reach gel fraction of more than $90 \%$ at $160{ }^{\circ} \mathrm{C}$. Both non-isothermal and isothermal curing investigation indicated that addition of DMAP as a catalyst is very efficient for accelerating the curing rate of ESO and DCA. The reaction between $-\mathrm{COOH}$ and epoxy is a nucleophilic addition reaction. DMPA as an organic base is able to react with $-\mathrm{COOH}$ to form $-\mathrm{COO}^{\ominus}$, which is more nucleophilic than carboxyl towards reaction with epoxy, thus reducing curing activation energy and increasing reaction rate. On the basis of previous studies ${ }^{20-22}$, we proposed the possible main reactions for curing of ESO and DCA in the presence of DMAP in Scheme 1.

We also monitored the curing of ESO/DCA with SA as an example DCA by using FT-IR spectra. For the curing of ESO and DCA, some functional groups such as carboxyl and epoxy will disappear and some others like hydroxyl and ester will generate. Therefore, FT-IR provides an efficient way to analyze the curing progress of the curing. Fig. 7 shows the FT-IR spectra of cured ESO/SA samples obtained by curing at $160{ }^{\circ} \mathrm{C}$ for different times. The peak of epoxy group at $910 \mathrm{~cm}^{-1}$ and the peak of $-\mathrm{COOH}$ at $1700 \mathrm{~cm}^{-1}$ decreased with reaction time, while a new broad peak of $-\mathrm{OH}$ at $3450 \mathrm{~cm}^{-1}$ and new peak of ester group at $1735 \mathrm{~cm}^{-1}$ generated and developed with time, confirming that the ring-opening of epoxy groups with $-\mathrm{COOH}$ happened, generating $-\mathrm{OH}$ and ester groups. It is noted that characteristic peaks of $-\mathrm{OH}$ and ester groups appeared even at 0 min, which indicate that partial curing of ESO and SA took place during premixing stage. When the reaction time prolonged to $120 \mathrm{~min}$, the 
characteristic peaks of epoxy and $-\mathrm{COOH}$ almost undetectable, indicating complete curing reaction, which is in accordance with the variation of gel fraction with reaction time.

\subsection{Thermal properties}

The glass transition temperature $\left(T_{\mathrm{g}}\right)$ of cured ESO/DCA samples were analyzed by DSC. Fig.8a shows the DSC heating scans of the samples. It is observed that the $T_{\mathrm{g}}$ shifted to lower temperature range with increasing chain-length of DCA. For example, $T_{\mathrm{g}}$ value for AA cured ESO is $-16{ }^{\circ} \mathrm{C}$, and those of SA and DA cured ESOs are -17.3 and $-21.6{ }^{\circ} \mathrm{C}$, respectively. With decrease in chain-length of DCA, crosslinking density increased, and thus free volume within the system decreased to restrict segment motion of the resultant product, which accounts for the increase in $T_{\mathrm{g}}$ with decreasing chain-length of DCA.

The thermal stability of the cured ESO/DCA samples were investigated by TGA. Fig. 8b shows the TGA curves of some typical samples recorded from room temperature to $550{ }^{\circ} \mathrm{C}$ at a heating rate of $10{ }^{\circ} \mathrm{C} / \mathrm{min}$ under $\mathrm{N}_{2}$ atmosphere. All samples showed similar thermal degradation behavior with good thermal stability. The initial thermal decomposition temperatures $\left(T_{5}\right.$, a temperature of $5 \%$ weight loss $)$ ranged from 340 to $370{ }^{\circ} \mathrm{C}$, and the maximum decomposition temperatures $\left(T_{\max }\right.$, corresponding to a maximum decomposition rate temperature) were all around $416{ }^{\circ} \mathrm{C}$. The chain-length and feed ratio of DCA/SEO showed some influences on the initial decomposition temperature of the samples but did almost not influence their $T_{\max }$. 


\subsection{Mechanical Properties}

Mechanical properties of DCA cured ESO samples were studied by tensile testing. Fig. 9 shows the stress-strain curves of some typical cured ESO/DCA samples. We can see from Fig. 9 that stress developed linearly with strain for all the samples. For the effect of chain-length of DCA on the tensile properties, both tensile strength and Young's modulus decreased while elongation at break showed uptrend with increasing chain-length of DCA, which is resulted from the decreased crosslinking density. The tensile strengths of AA, SA and DA cured ESO were $526 \pm 36,462 \pm 23$ and $453 \pm 41$ $\mathrm{KPa}$, respectively, the Young's moduli were $2.79 \pm 0.14,2.62 \pm 0.05$ and $2.30 \pm 0.16 \mathrm{MPa}$, respectively, and the elongations at break were $16.6 \pm 1.9,16.4 \pm 1.2$ and $20.6 \pm 2.3 \%$, respectively. It is noticed that the mechanical properties of the DCA cured ESO resin seemed insufficient for general plastic or elastomer application. However, they may find some applications in eco-friendly adhesive or coating without formaldehyde or some other toxic byproducts emission.

\section{Conclusions}

Fully sustainable polymers were synthesized by curing of ESO with biomass-derived DCAs with different carbon chain-lengths. The chain-length of DCAs affects the curing process significantly. Shorter-chained DCA showed higher reactivity and lower activation energy with respect to curing with ESO. The best molar ratio of $-\mathrm{COOH} / \mathrm{epoxy}$ was found at 0.7 for preparation of cured products with high degree of 
crosslinking. DMAP acted as an efficient catalyst to remarkably increase curing rate and decrease activation energy. Addition of $1.0 \mathrm{wt} \%$ DMAP decreased the activation energy by $7.5 \sim 11.6 \mathrm{~kJ} / \mathrm{mol}$ depending on the chain-length of DCA, and reduced the time for ESO/SA to achieve gel fraction of more than $90 \%$ from $8 \mathrm{hr}$ to $2 \mathrm{hr}$ during isothermal curing at $160{ }^{\circ} \mathrm{C}$. The chain-length of DCA also affected the physical properties of cured ESO/DCA samples. The glass transition temperature, tensile strength and Young's modulus decreased while elongation at break increased with increasing chain-length of DCA due to the decreased crosslinking density. All samples showed good thermal stability with $T_{5}$ of more than $340{ }^{\circ} \mathrm{C}$.

\section{Acknowledgements}

This work was supported by National Science Foundation of China (51673158) and

Fundamental Research Funds for the Central Universities (XDJK2015C022 and SWU115006).

\section{References}

(1) Z. Wang, X. Zhang, R. Wang, H. Kang, B. Qiao, J. Ma, L. Zhang, H. Wang, Synthesis and Characterization of Novel Soybean-Oil-Based Elastomers with Favorable Processability and Tunable Properties, macromolecules 45 (2012) 9010-9019.

(2) G.C. Liu, Y.S. He, J.B. Zeng, Q.T. Li, Y.Z. Wang, Fully Biobased and Supertough Polylactide-Based Thermoplastic Vulcanizates Fabricated by Peroxide-Induced 
Dynamic Vulcanization and Interfacial Compatibilization, Biomacromolecules 15 (2014) 4260-4271.

(3) A. Gandini, T. M. Lacerda, From monomers to polymers from renewable resources: Recent advances, Prog. Polym. Sci. 48 (2015) 1-39.

(4) R. T. Zeng, W. Hu, M. Wang, S. D. Zhang, J. B. Zeng, Morphology, rheological and crystallization behavior in noncovalently functionalized carbon nanotube reinforced poly(butylene succinate) nanocomposites with low percolation threshold, Polym. Test. 50 (2016) 182-190.

(5) D. Yuan, C. Xu, Z. Chen, Y. Chen, Crosslinked bicontinuous biobased polylactide/natural rubber materials: Super toughness, "net-like"-structure of NR phase and excellent interfacial adhesion. Polym. Test. 38 (2014) 73-80.

(6) F. S. Guner, Y. Yagci, A. T. Erciyes, Polymers from triglyceride oils, Prog. Polym. Sci. 31 (2006) 633-670.

(7) M. A. R. Meier, J. O. Metzger, U. S. Schubert, Plant oil renewable resources as green alternatives in polymer science, Chem. Soc. Rev. 36 (2007) 1788-1802.

(8) B. Cortese, M. H. J. M. de Croon, V. Hessel, High-temperature epoxidation of soybean oil in flow-speeding up elemental reactions wanted and unwanted, Ind. Eng. Chem. Res. 51 (2012) 1680-1689.

(9) C. Bueno-Ferrer, M. C. Garrigos, A. Jimenez, Characterization and thermal stability of poly(vinyl chloride) plasticized with epoxidized soybean oil for food packaging. Polym. Degrad. Stab. 95 (2010) 2207-2212. 
(10)S. Vijayarajan, S. E. M. Selke, L. M. Matuana, Continuous Blending Approach in the Manufacture of Epoxidized SoybeanPlasticized Poly(lactic acid) Sheets and Films, Macromol. Mater. Eng. 299 (2014) 622-630.

(11)D. Yang, X. Peng, L. Zhong, X. Cao, W. Chen, X. Zhang, S. Liu, R. Sun, "Green" films from renewable resources: Properties of epoxidized soybean oil plasticized ethyl cellulose films, Carbohydr. Polym. 103 (2014) 198-206.

(12)A. Li, K. Li, Pressure-Sensitive Adhesives Based on Epoxidized Soybean Oil and Dicarboxylic Acids, ACS Sustain. Chem. Eng. 2 (2014) 2090-2096.

(13)A. Rucigaj, B. Alic, M. Krajnc, U. Sebenik, Investigation of cure kinetics in a system with reactant evaporation: Epoxidized soybean oil and maleic anhydride case study, Eur. Polym. J. 52 (2014) 105-116.

(14)Q. Luo, M. Liu, Y. Xu, M. Ionescu, Z. S. Petrović, Thermosetting Allyl Resins Derived from Soybean Oil, Macromolecules 44 (2011) 7149-7157.

(15) Y.H. Lia, X. S. Sun, Synthesis and characterization of acrylic polyols and polymers from soybean oils for pressure-sensitive adhesives, RSC Adv. 5 (2015) 44009-44017.

(16)J. Dai, S. Ma, Y. Wu, L. Han, L. Zhang, J. Zhu, X. Liu, Polyesters derived from itaconic acid for the properties and bio-based content enhancement of soybean oil-based thermosets, Green Chem. 17 (2015) 2383-2392.

(17)C. Zhang, Y. Li, R. Chen, M. R. Kessler, Polyurethanes from solvent-free vegetable oil-based polyols, ACS Sustain. Chem. Eng. 2 (2014) 2465-2476. 
(18)R. Ghoreishi, G.J. Suppes, Modeling of toluene sulfonic acid catalyzed oxide addition reaction for soy-based polyol, Ind. Eng. Chem. Res. 54 (2015) 91-99.

(19)C. Zhang, M. R. Kessler, Bio-based Polyurethane Foam Made from Compatible Blends of Vegetable-Oil-based Polyol and Petroleum-based Polyol. ACS Sustain. Chem. Eng. 3 (2015) 743-749.

(20)C. Ding, P. S. Shuttleworth, S. Makin, J. H. Clark, A. S. Matharu, New insights into the curing of epoxidized linseed oil with dicarboxylic acids, Green Chem. 17 (2015) 4000-4008.

(21)S. G. Tan, W. S. Chow, Curing Characteristics and Thermal Properties of Epoxidized Soybean Oil Based Thermosetting Resin, J. Am. Oil Chem. Soc., 88 (2011) 915-923.

(22)S. G. Tan, Z. Ahmad, W. S. Chow, Relationships of cure kinetics and processing for epoxidized soybean oil bio-thermoset, Ind. Crop. Prod. 43 (2013) 378-385.

(23)N. Supanchaiyamat, P. S. Shuttleworth, A. J. Hunt, J. H. Clark, A. S. Matharu, Bio-based thermoset composites from epoxidised linseed oil and expanded starch, Green Chem. 14 (2012) 1759-1765.

(24)H. E. Kissinger, Reaction Kinetics in Differential Thermal Analysis, Anal. Chem. 29 (1957) 1702-1706. 
Table 1. non-isothermal curing behavior and activation energy for curing reaction of ESO and DCAs.

\begin{tabular}{llll}
\hline Sample & $T_{\mathrm{p}}\left({ }^{\circ} \mathrm{C}\right)$ & $\Delta H_{\mathrm{c}}(\mathrm{J} / \mathrm{g})$ & $\Delta E_{\mathrm{a}}(\mathrm{kJ} / \mathrm{mol})$ \\
\hline ESO/AA & $200.2 \pm 1.8$ & $245.6 \pm 2.5$ & $82.8 \pm 1.1$ \\
ESO/AA/DMAP-0.5 & $188.6 \pm 0.9$ & $248.4 \pm 5.3$ & $81.1 \pm 0.6$ \\
ESO/AA/DMAP-1.0 & $178.5 \pm 1.6$ & $243.4 \pm 4.7$ & $71.2 \pm 0.7$ \\
ESO/SA & $206.6 \pm 1.0$ & $242.5 \pm 2.3$ & $83.5 \pm 2.5$ \\
ESO/SA/DMAP-0.1 & $203.8 \pm 2.2$ & $232.5 \pm 2.8$ & - \\
ESO/SA/DMAP-0.3 & $197.1 \pm 1.6$ & $238.9 \pm 1.9$ & - \\
ESO/SA/DMAP-0.5 & $189.6 \pm 1.2$ & $235.8 \pm 3.2$ & $80.6 \pm 1.7$ \\
ESO/SA/DMAP-1.0 & $178.2 \pm 0.7$ & $240.9 \pm 1.7$ & $76.0 \pm 0.6$ \\
ESO/SA/DMAP-3.0 & $172.5 \pm 1.5$ & $237.8 \pm 3.6$ & - \\
ESO/DA & $209.6 \pm 3.3$ & $232.5 \pm 1.8$ & $85.4 \pm 0.9$ \\
ESO/DA/DMAP-0.5 & $191.4 \pm 1.2$ & $222.7 \pm 3.3$ & $76.0 \pm 0.5$ \\
ESO/DA/DMAP-1.0 & $180.5 \pm 1.3$ & $229.3 \pm 5.6$ & $74.1 \pm 0.7$ \\
\hline
\end{tabular}




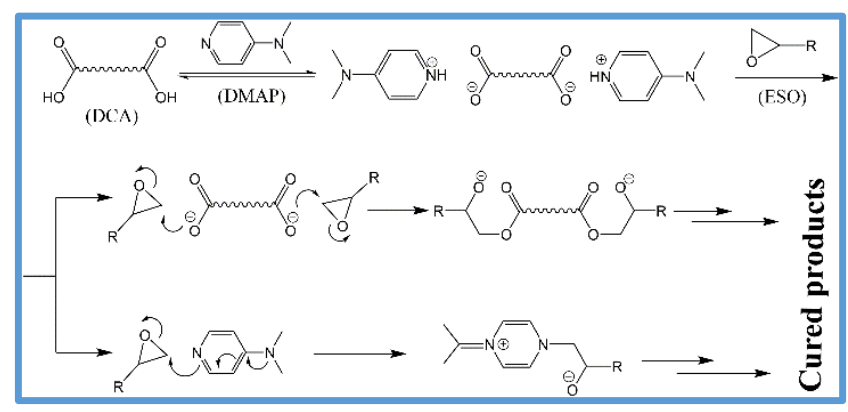

Scheme 1. Possible main reactions for curing of ESO and DCA in the presence of DMAP. 


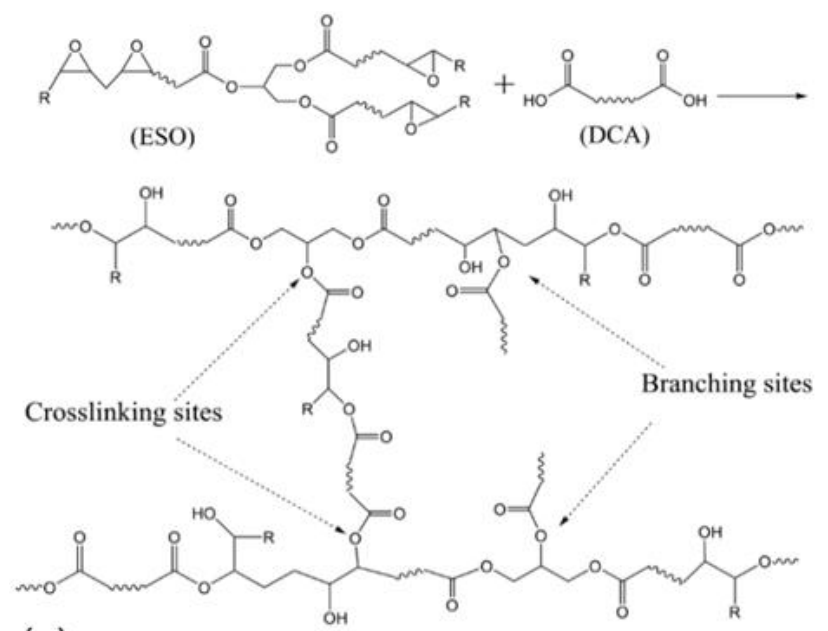

(a)

(Cured product)

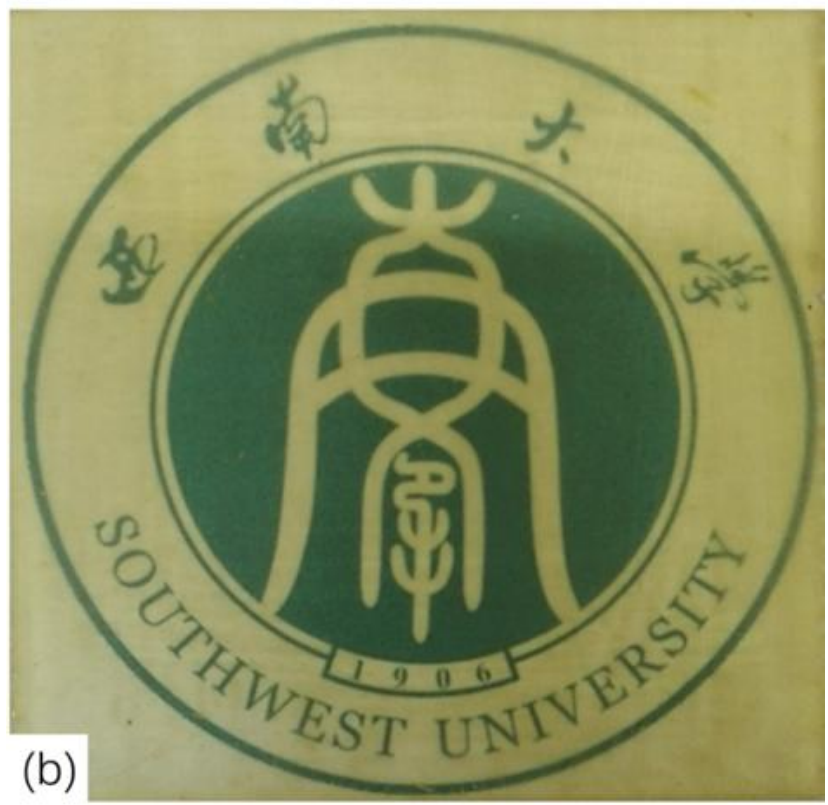

Fig. 1. Proposed curing reaction between ESO and DCA (a) and the digital photo of prepared transparent film (b). 


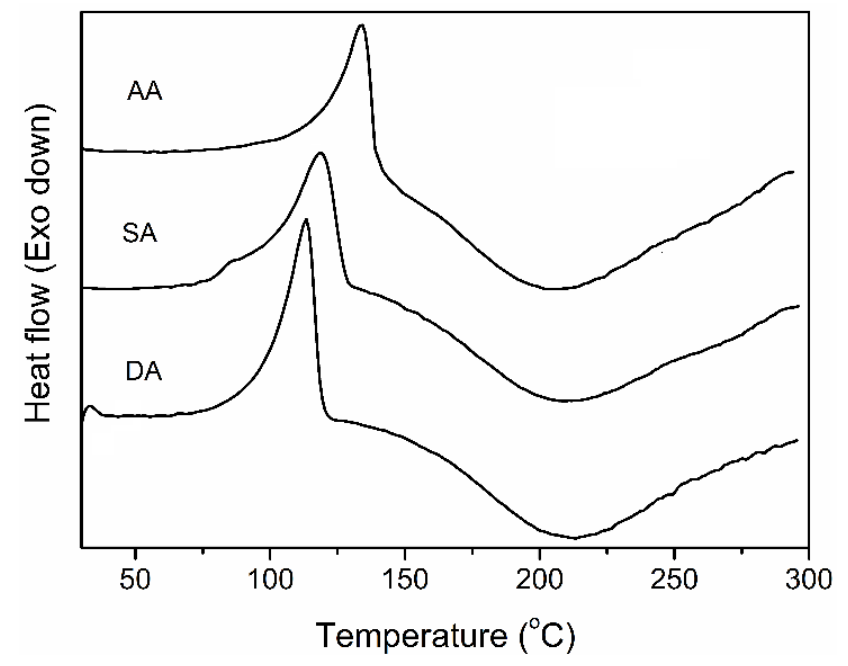

Fig. 2. DSC heating scans for non-isothermal curing behaviours of ESO cured with different DCAs. 


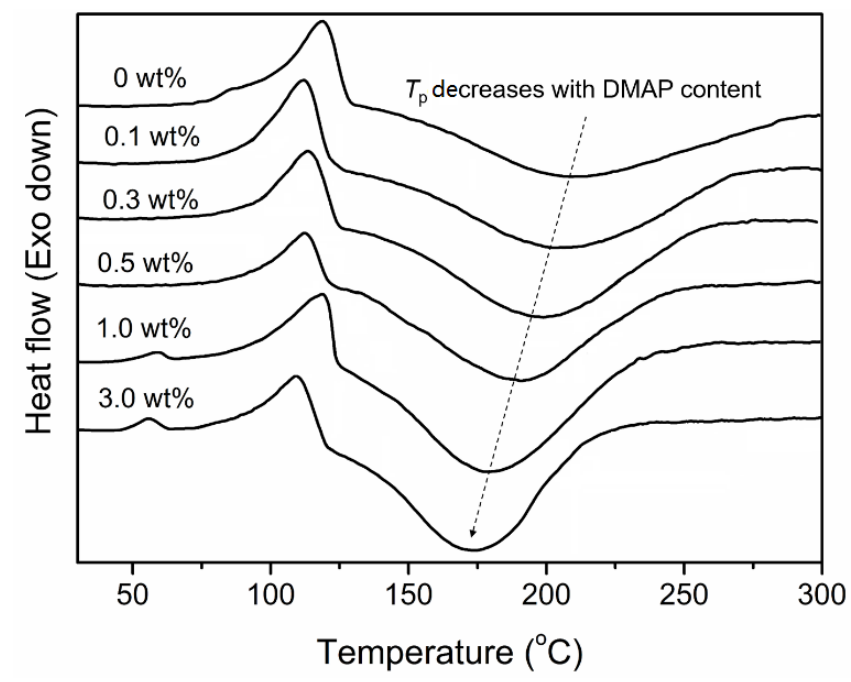

Fig. 3. DSC heating scans for non-isothermal curing behaviours of ESO cured by SA with different content of DMAP. 


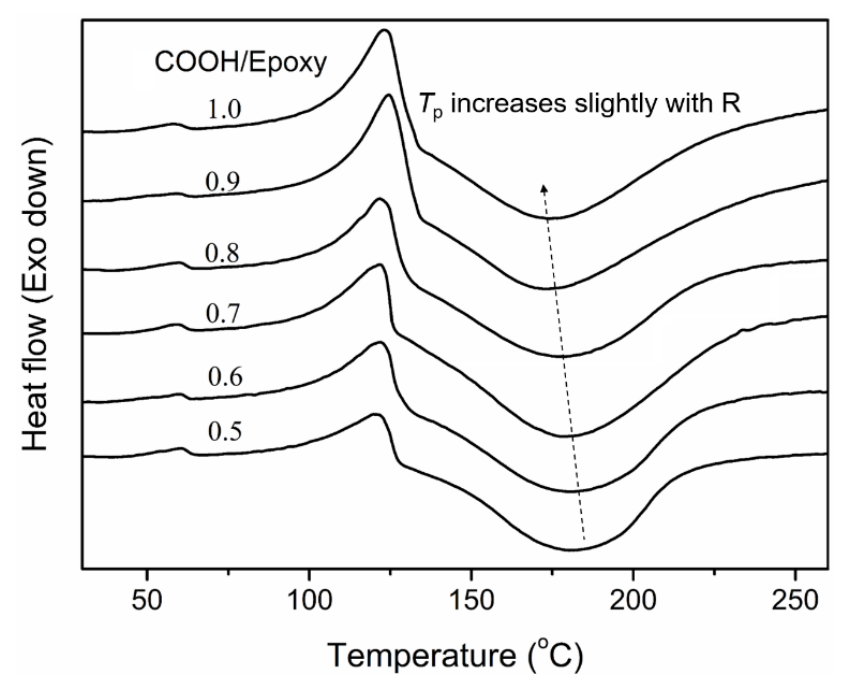

Fig. 4. DSC heating sans for non-isothermal curing behaviors of ESO with SA at different - $\mathrm{COOH} / \mathrm{epoxy}$ molar ratios. 

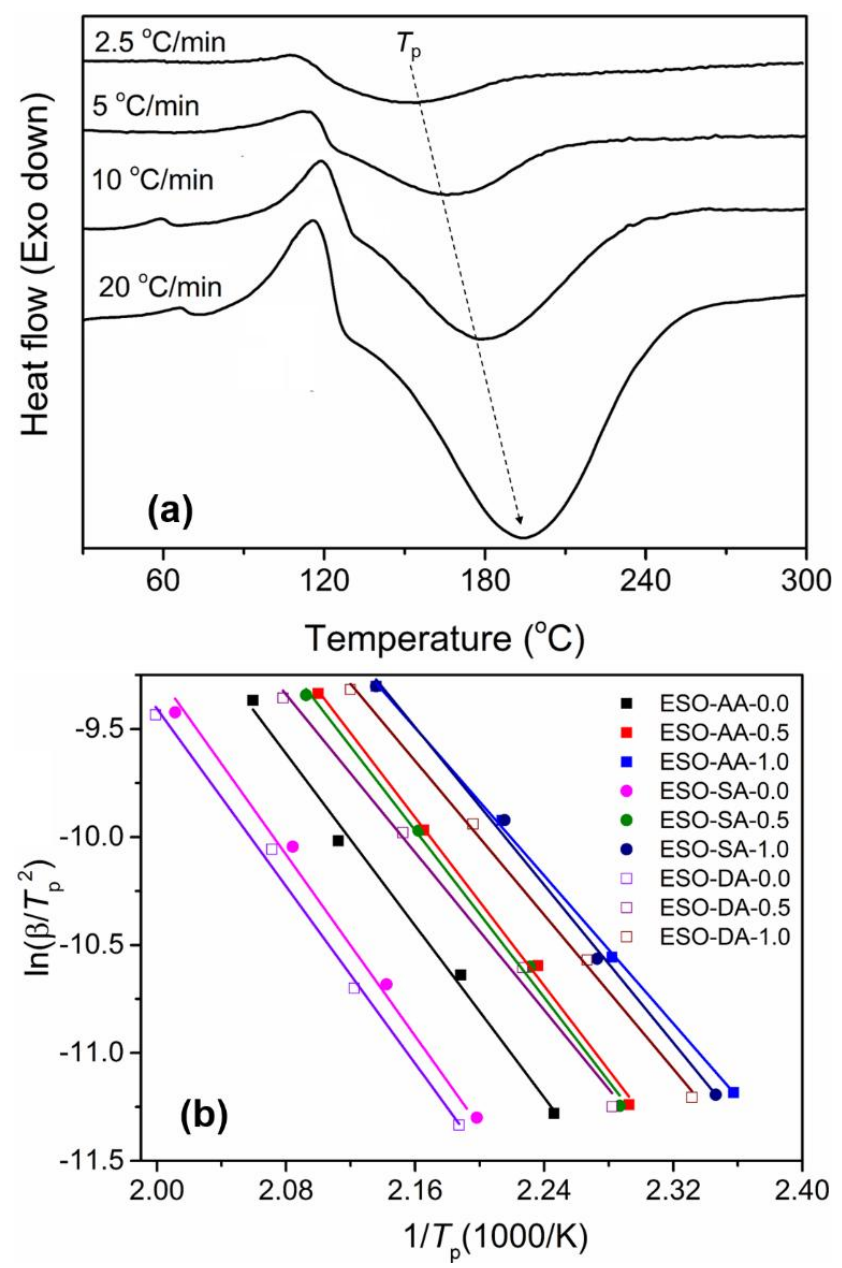

Fig. 5. Typical dynamic DSC heating scans of ESO/SA/DMAP-1.0 with various heating rates (a) and Kissinger plot for determination of activation energy (b). 

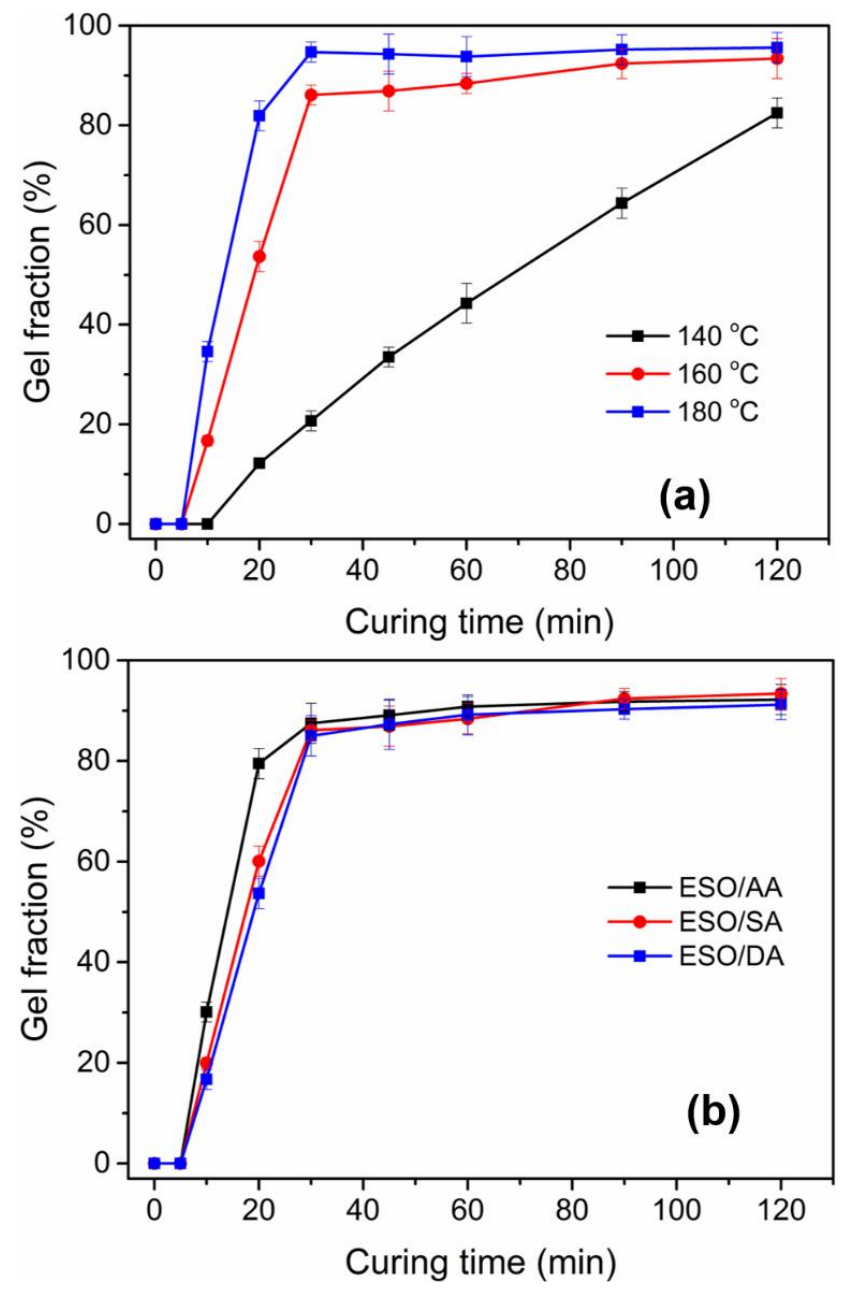

Fig. 6. Development of gel fraction of cured ESO/SA/DMAP-1.0 with time at different isothermal curing temperatures (a) and development of gel fraction with time for ESO cured with different DCAs at $160{ }^{\circ} \mathrm{C}$. 


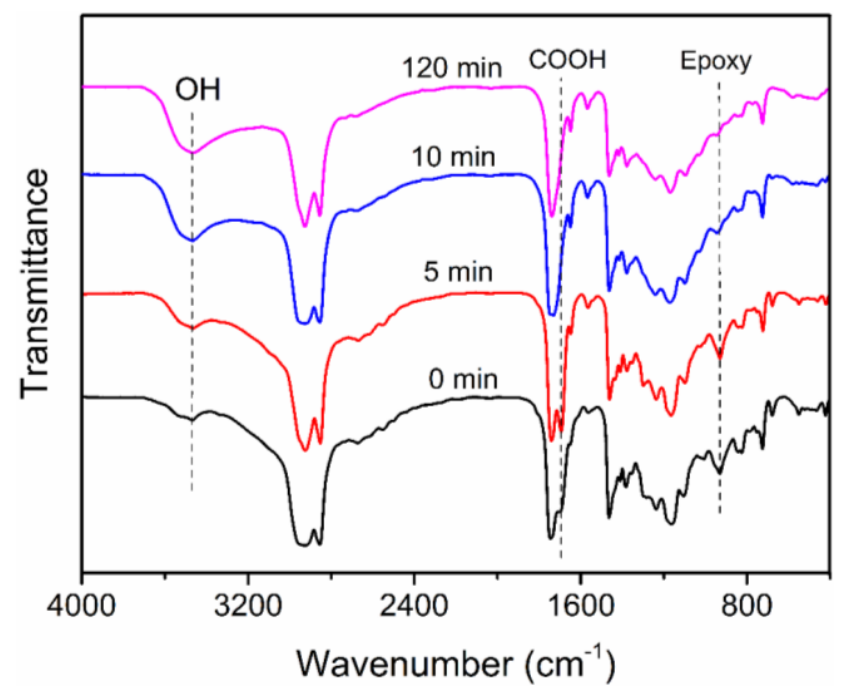

Fig. 7. FT-IR spectra of cured ESO/SA samples obtained by isothermal curing at $160{ }^{\circ} \mathrm{C}$ for various times. 

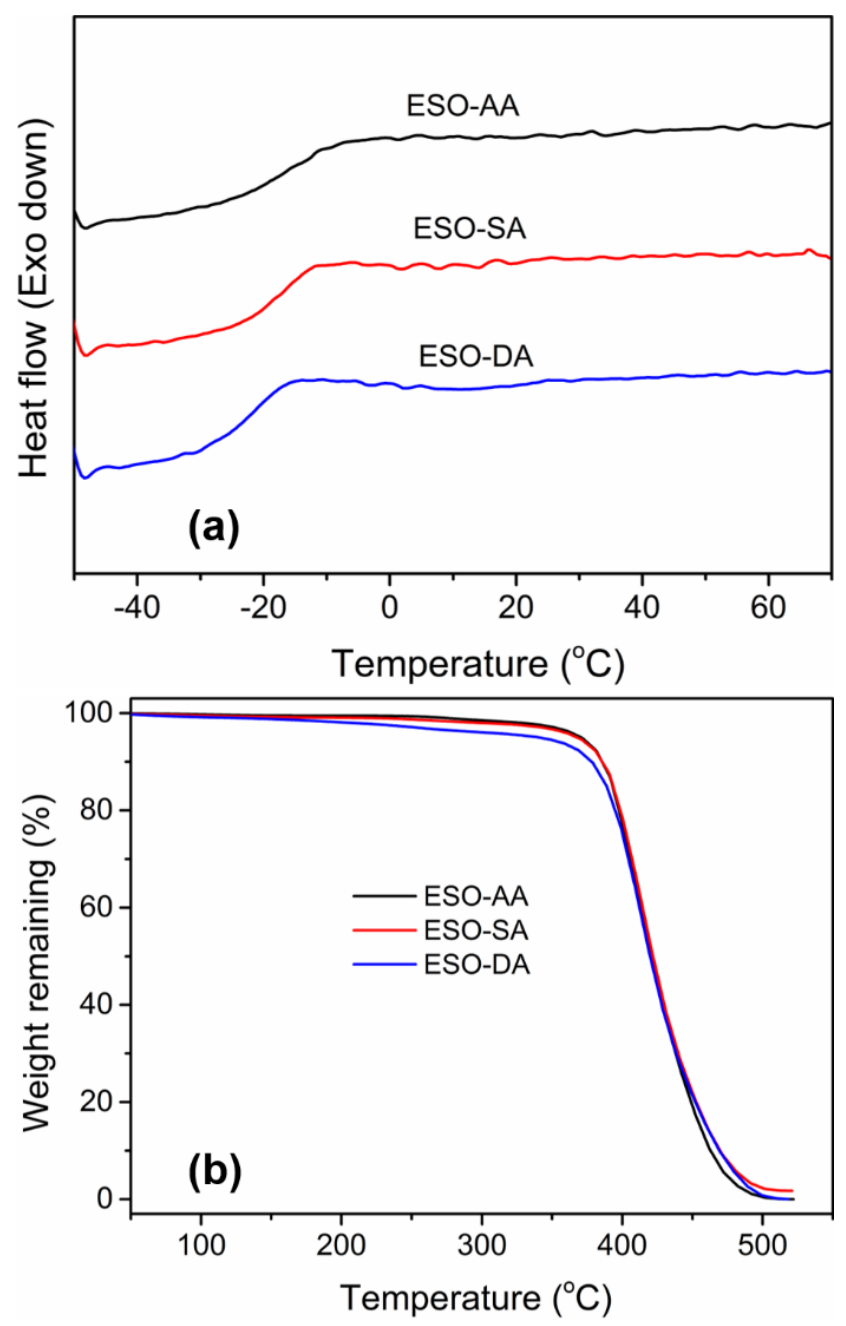

Fig. 8. DSC heating scans (a) and TGA curves (b) of different chain-length DCAs cured ESO. 


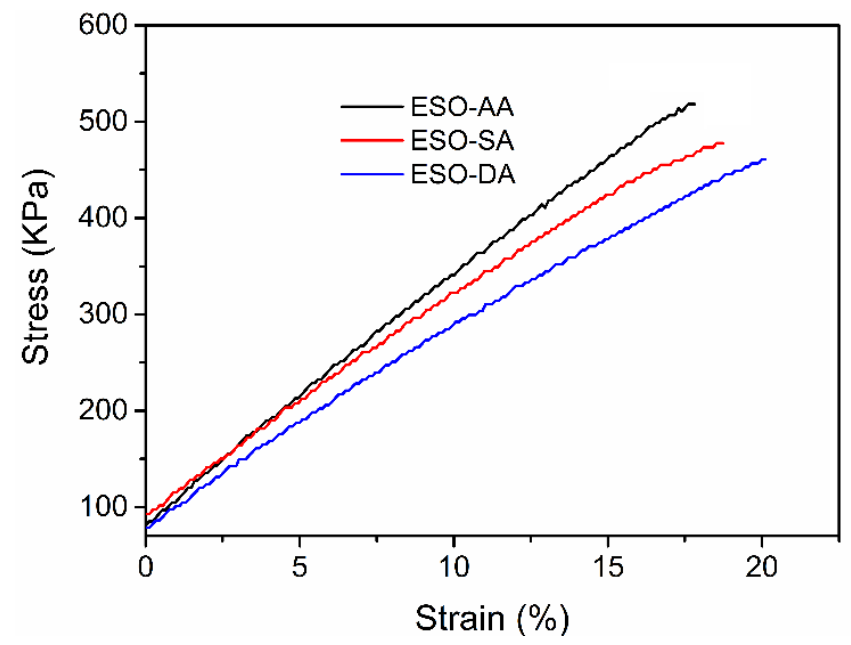

Fig. 9. Stress-strain curves of typical cured ESO/DCA samples. 